\title{
GRAN EMPRESA, SUBDESARROLLO Y DETERIORO AMBIENTAL
}

Hugo Ordóñez Salazar', Ramón Pichs Madruga

\section{LA DINÁMICA ECONÓMICA}

En oposición a la distorsionada e interesada prédica neoliberal, el sistema económico vigente, de acuerdo a los planteamientos básicos de la Teoria Económica, está motorizado por el mercado de competencia imperfecta, signado en la fase superior del capitalismo, por la presencia monopólica (polipolios, oligopolios. monopsonios, etc.) de grandes empresas transnacionales, generalmente sociedades anónimas, que son organizaciones accionarias, de capital, que actuando en función del principio de racionalidad de la economía (irracionalidad según Maurice Godellier), sólo buscan maximizar sus beneficios, su tasa de expoliación y plusvalia, única garantía de incremento de su tasa de acumulación; para lo cual, es condición sine-quanon, minimizar sus costos de producción; objetivos que sólo pueden obtenerlos explotando el capital humano (factor trabajo) y depredando el factor naturaleza, principalmente de los paises subdesarrollados.

Seguidamente, en relación al tema que estamos tratando, señalaremos escuetamente, algunas de las características, más sustantivas, de la gran empresa transnacional:

- Utiliza tecnología de punta (la más avanzada en el momento actual), intensiva en el uso del factor capital, que por tenerlo en abundancia, resulta ser el más baratoen los países capitalistas desarrollados; realidaddiametralmente diferente a nuestra dotación factorial; lo que nos plantea el problema de la convertibilidad de nuestros factores y del aparato productivo, de la reconversión industrial, de la adecuación tecnológica y d e creación de tecnologia intermedia, de acuerdo a nuestras limitaciones, posibilidades e intereses, para

1. Economista

2. Integrante del Centro de Investigación de la Economia Mundial - LA HABANA, CUBA. operar con funciones productivas que privilegien el uso intensivo de nuestros factores más abundantes, e incluso ociosos (mano de obra y recursos naturales), en campos en los que tenemos incuestionables ventajas comparativas $y$ competitivas, como en la biotecnologia, genética, etc., en un marco de programación e integración continental, que asegure nuestra presencia, a nivel de toma de decisiones, en la escena mundial.

Debemos remarcar, que la renovación tecnológica incesante, cada vez más concentradora de capital, en los paises capitalistas avanzados, es la condición necesaria, para tener éxito en la competencia intermonopólica.

- La producción en gran escala, para megamercados, vía el efecto demostración y la rapidez de los medios de comunicación, permite su mundialización, posibilitando disminuir sus costos de producción, que frente a precios constantes o crecientes, le significan un aumento del beneficio total.

- Anula lo que debería ser la inmaculada "Soberanía del Consumidor", puesto que impone precios y calidad de los productos, generando consumos residuales e irracionales, negando totalmente al pontificado "libre mercado", en el que deberian operar espontánea y libremente las fuerzas de la oferta y demanda.

\section{EL PODER ECONÓMICO Y EL PODER POLITICO}

Considerando que: "la política es la expresión concentrada de la economia", debemos colegir que ambos poderes constituyen un todo único. Siendo la gran empresa monopólica transnacional, Ia representación máxima del sistema capitalista en su fase imperial, constituye un poderoso grupo de presión que concentra el poder económico y el poder político, puestos al servicio crematístico de incrementar su rentabilidad económica. 
Tal vinculación se comprueba fácilmente, al observar la extrema sensibilidad de sus Gobiernos (grupos económicos y organismos financieros internacionales que controlan), respecto a problemas generados por esas empresas en cualquier pais del tercer mundo, verbigracia, el caso peruano, en que por una soberana decisión gubernamental de expropiar y estatizar el petróleo, explotado por la International Petroleum Company, se aplicó en la década del 70, la Ilamada Enmienda Hickenlooper, cortándole todo tipo de asistencia económica financiera y, últimamente, la Banca Privada externa, acreedora, con la aquiescencia del poder oficial, impone como condición para la renegociación de la deuda del pais, el previo pago a una empresa norteamericana de una transacción, probadamente corrupta, en la compra de dos barcos (chatarra) Mantaro y Pachitea.

De esa manera, se evidencia, cómo el Gobierno norteamericano, es la representación política de los grupos que controlan su economia, y que en nuestro continente, tipificado como el "patio trasero de los Estados Unidos", asi como en el resto del mundo subdesarrollado, su interés alcanza hasta donde alguna de sus empresas tengan invertido un dolar americano.

Precisamente, el uso y abuso de poder, posibilita que realicen en nuestros países, actividades productivas que ya han sido prohibidas o limitadas en sus territorios, por su carácter contaminante y por generar apreciables deseconomias externas; es por ello que, en la medida que los estandares ambientales se tornan más exigentes en los paises desarrollados, para preservar su equilibrio ecológico y un medio ambiente saludable, tienden a transferir, crecientemente, sus empresas nocivas y tecnologias sucias a nuestros países, pretendiendo incluso, convertirnos en meros basureros atómicos, deteriorando gravemente el aire, mar y tierra de nuestro ecosistema; proceso intenso y grave que en términos de una certera y justa evaluación económica, ha generado una DEUDA ECOLOGICA que supera largamente a la llamada DEUDA EXTERNA, por lo que legitimamente, el Norte le tiene una importante deuda pendiente al Sur.

\section{SUBDESARROLLO, POBREZA Y DETERIORO AMBIENTAL}

A mediados de la década del ochenta, se asiste a un proceso de internacionalización del debate en torno al vínculo entre medio ambiente y desarrollo, que tiene importantes dimensiones politicas, económicas, tecnológicas, sociales y humanas. Ultimamente, se han hecho más evidentes algunos de los problemas ambientales de alcance global que preocupan profundamente a la humanidad, tales como: el agotamiento de la capa de ozono, el "efecto de invernadero", la pérdida de diversidad biológica, la contaminación urbana, de los mares y zonas costeras, el tráfico transfronterizo de deshechos peligrosos y el deterioro ambiental asociado a las condiciones de subdesarrollo y pobreza en que viven las tres cuartas partes de la población mundial.

Sin dejar de considerar la profunda decepción que dejó en muchos la falta de compromisos concretos de la Conferencia de Naciones Unidas sobre Medio Ambiente y Desarrollo (CNUMAD), Brasil/92, debe señalarse que posiblemente, el principal logro de esa reunión fue el reconocimiento de que la protección ambiental y el desarrollo económico requieren soluciones globales. Por primera vez se logró consenso, al más alto nivel gubernamental, para adoptar un nuevo enfoque sobre el desarrollo, donde la erradicación de la pobreza y la protección del medio estén estrechamente vinculadas.

Un análisis integral de la relación entre medio ambiente y desarrollo, en el actual contexto internacional, debe tomar en consideración la deuda ecológica del mundo desarrollado y la persistencia de un circulo vicioso entre subdesarrollo, pobreza y deterioro ambiental. Por un lado, los patrones de desarrollo seguidos por los países industrializados han ocasionado los mayores daños al ecosistema, sobre todo si se analiza el deterioro del medio ambiente desde una perspectiva histórica. Los paises desarrollados, con la quinta parte de la población mundial, reciben las cuatro quintas partes del ingreso mundial y utilizan alrededor de las cuatro quintas partes de los recursos naturales $(70.0 \%$ de la energía, $75.0 \%$ de los metales y $85.0 \%$ de la madera).

Especificamente, los paises de la Organización para la Cooperación y el Desarrollo Económico (OCDE) absorben entre el $40.0 \%$ y el $60.0 \%$ del consumo mundial de importantes producciones de origen mineral, tales como los combustibles fósiles $(43.0 \%)$, petróleo $(50.0 \%)$, acero $(40.0 \%)$, aluminio $(58.0 \%)$, cobre $(58.0 \%)$, plomo $(55.0 \%)$, niquel $(59.0 \%)$, estaño $(53.0 \%)$ y zinc $(46.0 \%)$. Consecuentemente, estos estados aportan más del $50.0 \%$ de las emisiones de gases del efecto de invernadero, incluída la producción de alrededor del $90.0 \%$ de los clorofluorocarbonos -que son además 
los principales causantes del deterioro de la capa de ozono estratosférico-, emiten la mayor parte de los gases que provocan las precipitaciones ácidas, es decir, $40.0 \%$ del dióxido de azufre y $54.0 \%$ de los óxidos de nitrógeno, y generan el $68.0 \%$ de los deshechos industriales.

Debe destacarse, la enorme responsabilidad de las empresas transnacionales en el deterioro de nuestro medio ambiente, puesto que controlan la cuarta parte de los activos productivos mundiales, el $70.0 \%$ del comercio internacional, el $80.0 \%$ de la tierra dedicada a los cultivos de exportación y la mayor parte de las innovaciones tecnológicas realizadas a nivel internacional; además, son responsables, por ejemplo, de más del $50.0 \%$ de las emisiones globales de gases del efecto de invernadero, en tanto controlan la mitad de la producción petrolera mundial. prácticamente toda la producción de vehículos de carretera fuera de Europa del Este, la mayor parte de los clorofluorocarbonos producidos, así como una parte significativa de la generación y uso de electricidad.

Por otro lado, la pobreza ha sido identiticada como uno de los principales obstáculos para un desarrollo ambientalmente seguro, ya que la mayoría de los pobres $(60.0 \%)$ viven en áreas ecológicamente vulnerables. En las naciones subdesarrolladas, según datos de 1991-1992, alrededor de 1,300 millones de personas carecen de suministros de agua potable, 1,000 millones están excluídos de los servicios de salud y 1,900 millones no tienen acceso a los servicios de saneamiento; aproximadamente 17 millones de habitantes mueren al año por contraer enfermedades infecciosas y parasitarias tales como la diarrea, la malaria y la tuberculosis; unos 800 millones de personas presentan déficit alimentario; casi mil millones de personas $(35.0 \%$ de la población adulta) son analfabetos; un tercio de la población total (unos 1,300 millones de seres humanos) se hallan en condiciones de absoluta pobreza; 900 millones de personas viven en áreas afectadas por la desertificación y podría haber 14 millones de "refugiados ambientales ".

En el período 1990 - 2030 es probable que la población mundial crezca en 3,700 millones de habitantes y el $90.0 \%$ de ese crecimiento tendrá lugar en las naciones subdesarrolladas. Si el Sur repitiera las pautas de desarrollo del Norte, se requiriria 10 veces las existencias actuales de minerales y al cabo de 40 años estas necesidades se duplicarian al doblarse la población.
En muchas ocasiones, estas poblaciones, que viven en condiciones de absoluta pobreza, no tienen otra alternativa que depredar el medio ambiente para intentar sobrevivir y, como se trata de economias subdesarrolladas y altamente dependientes de las exportaciones de productos básicos, al erosionarse el medio se afectan sensiblemente las principales fuentes de ingresos exportables. Se estima que alrededor del $45.0 \%$ de los ingresos por exportación del Tercer Mundo corresponde a los productos básicos y en el caso de la región africana esta proporción asciende al $90.0 \%$.

En este contexto, las preocupaciones ambientales inmediatas de los paises desarrollados difieren considerablemente con relación a las de los paises subdesarrollados. En los estados industrializados, donde lo que está en peligro es la calidad de la vida, existe una preocupación creciente por los efectos, a más largo plazo, de fenómenos tales como el deterioro de la capa de ozono y el calentamiento global, entre otros; sin embargo, para las naciones subdesarrolladas, donde lo que está en peligro es el derecho a una vida digna, las principales preocupaciones están relacionadas con problemas tan acuciantes como la calidad del agua y la protección del suelo.

En franco contraste con las aspiraciones de equidad y justicia en las relaciones internacionales, el nuevo orden mundial, que se va imponiendo en las esferas económica y politica, obstaculiza el avance en materia de integración, entre medio ambiente y desarrollo. Este llamado nuevo orden mundial, liderado por los Estados Unidos, ha sido definido por algunos autores como un sistema unipolar en el orden político - militar y tripolar en el orden económico, que tiene, entre otros principios de funcionamiento, un caracter asimétrico y el hecho de ser impuesto por uno o algunos paises al resto de la comunidad internacional.

Resulta importante llamar la atención sobre los riesgos asociados a algunos de los instrumentos que se han propuesto a nivel internacional, para hacer cumplir los acuerdos de la Cumbre de Rio de Janeiro; al respecto, en el Mensaje de Cuba a esa Conferencia se señalan, los posibles efectos negativos para los paises subdesarrollados, de un sistema internacional de permisos de emisión negociables, si se tiene en cuenta la enorme brecha socio - económica existente entre el Norte y el Sur. Recientemente, los paises desarrollados han estado promoviendo una fórmula conocida como "Implementación Conjunta" (Joint 
Implementation), referida a arreglos conjuntos entre paises para hacer cumplir los compromisos internacionales de la Convención Marco de Naciones Unidas sobre Cambio Climático, bajo criterios de "Costo Eficiencia"; mecanismo que tiene como riesgo fundamental para su aplicación entre los paises desarrollados y subdesarrollados, la posibilidad de que los primeros, como siempre, no cumplan con sus compromisos, tanto en materia de reducción de la contaminación en sus propios territorios, como en lo relacionado a la transferencia de recursos financieros adicionales y de tecnología hacia los paises subdesarrrollados.

Asi las cosas, el avance del Tercer Mundo por la senda de un desarrollo ambientalmente seguro, estaría condicionado, en lo fundamental, por el éxito que se tenga en el mejoramiento del entorno económico internacional en que estamos insertados.

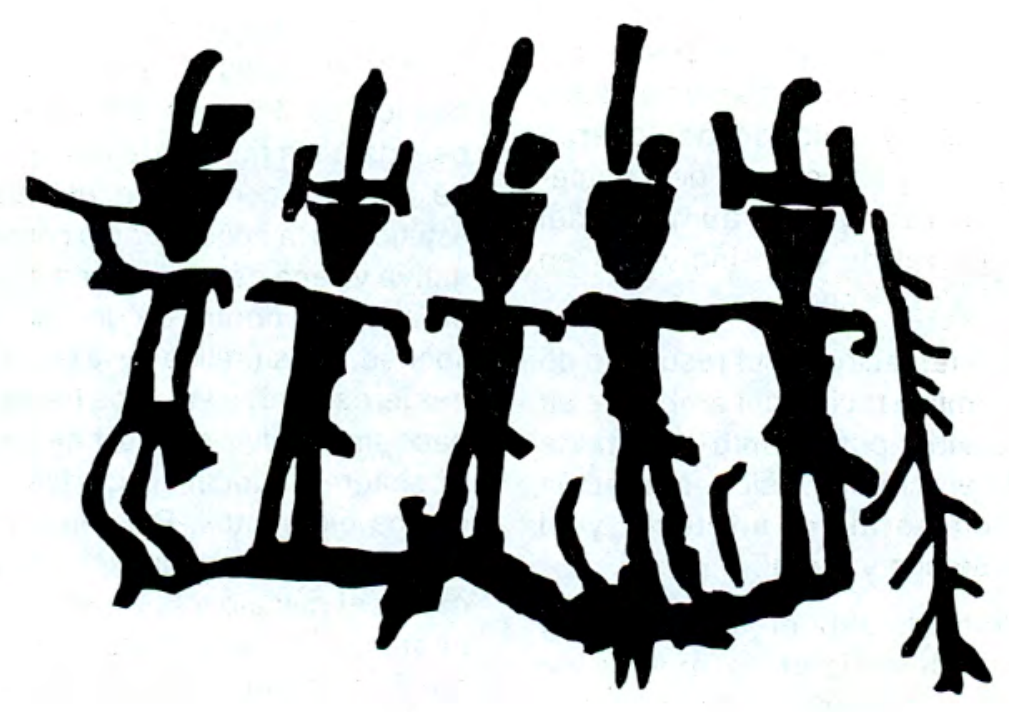




\section{TALLER DE INVESTIGACIÓN CIENTÍFICO DEPORTIVA}

\author{
William Bartesaghi Aste, Pedro Tejada \\ Monroy, Eloina Tejada Munroy. \\ Facultad de Ciencias de la Educacion
}

La expresión o palabra Deporte puede ser considerada como un conjunto de reglas destinadas al mantenimiento, control y cuidado del cuerpo humano; empero, es un arte y una ciencia de carácter biopsicopedagógico que persigue el aumento del potencial biológico y moral de todo individuo en relación a su capacidad y eficiencia.

Como se sabe, educación es el resultado de las influencias que el hombre recibe del ambiente en que se desenvuelve su vida: por lo tanto, el deporte es un medio preponderantemente físico, que actúa sobre el desarrollo corporal, el intelecto y el desenvolvimiento psicológico y moral.

Cuando hablamos de deporte se debe tener en cuenta la particular diversidad de medios de instalación que, para su eficaz aplicación, requiera. Toda educación deportiva está basada en la capacidad del hombre de desplazarse en el fenómeno biológico del movimiento.

La ciencia ha comprobado que todo acto, hasta el más simple movimiento de una mano se realiza a través de un proceso neuromuscular, con intervención de vía refleja cuando es involuntario; también se ha demostrado que las tres cuartas partes de la masa cerebral poseen funciones motrices. EI juego, por citar uno de los agentes del deporte, se resuelve ineludiblemente por medio de procesos semejantes, de naturaleza mental y física.

La planificación de cualquier táctica de defensa y de ataque, por ejemplo, es tarea de orden intelectual, que luego se transforma en acto físico.
De manera concomitante, la moral se ejercita en el cumplimiento de las normas del juego establecidas y la esfera psíquica se amplia con las vivencias que aporta el trato entre los individuos, en relación de la solidaridad, rivalidad y espiritu de equipo.

La antigua escuela pedagógica, caracterizada por la inflexibilidad de los programas, fue seguida a comienzos del siglo actual por una educación física basada en la rigidez de una gimnasia de corte militar, de acuerdo con las necesidades bélicas y el gusto estético de la época. Por lo contrario, la escuela nueva, activa y llena de vida, Ilama a la participación integral de alumnos dentro de un clima en el que imperan la libertad, la naturalidad y la camaraderia. La pedagogia moderna vuelve sus ojos hacia la Grecia clásica y no hace sino revivir el ideal helénico: música y gimnasia. El sistema educativo de los griegos se basaba en dichos elementos. Por música entendian el estudio de la danza, la recitación, la poesía, la oratoria, el canto, el cálculo y la música propiamente dicha; por gimnasia, el desarrollo armonioso del cuerpo por medio de la ejercitación física, el régimen alimentario, los masajes y la vida sana al aire libre. Ambos métodos estaban estrechamente unidos pues la gimnasia se realizaba al son de la música. Los helenos se hallaban convencidos de que la salud corporal y el control del carácter operaban un efecto favorable en las cualidades intelectuales y morales. Han debido transcurrir veinte siglos para reencontrar en la época contemporánea el justo equilibrio entre la educación intelectual y la formación física y moral.

La educación deportiva es fundamentalmente una actitud, una manera de vivir, un punto de vista e incidentalmente una técnica y una habilidad particular lo que significa que es el logro de la salud, la estabilidad del sistema nervioso y el control emocional. 


\section{MÉTODO COMPUTARIZADO PARA AUMENTAR VOCABULARIO DE INGLÉS INTERMEDIO CON FINES DE CAPACITACIÓN}

Ricardo ( Carranza De La Torre Facultad de' Ing. Pesquera

En la actualidad, el conocimiento de una segunda lengua es imprescindible para un desempeño más eficiente y actualizado en todos los campos de la actividad profesional y no profesional. El idioma inglés ocupa la primera preferencia dada su universalidad, impulsada por los paises para los cuales es lengua madre.

Se tiene un nivel intermedio de inglés cuando se han dedicado a su estudio un aproximado de 300 horas y se conocen alrededor de 600 palabras. Esto permite leer ciertos temas especializados si se conocen las palabras de función y contenido correspondientes. Este es el caso de los docentes universitarios, muchos estudiantes y otras tantas personas que pertenecen a diversas comunidades intelectuales, profesionales y de todo tipo.

El problema que la mayoria de ellos enfrenta, en su afán de mantener y mejorar su conocimiento y manejo de inglés, es el tiempo que disponen para este propósito, simplemente no pueden hacer uso de métodos existentes porque son consumidores de tiempo.

Muchas de las becas de capacitación y perfeccionamiento que existen en oferta cada año, se pierden precisamente por falta de dominio del idioma inglés. Por otra parte, el que hace uso de becas en el extranjero debe necesariamente avanzar del modo más rápido en la tarea de incrementar el número de palabras que conoce para poder comunicarse.

Se necesitan de 1500 a 2000 palabras para lograr fluidez minima al hablar, leer corrido y redactar satisfactoriamente en una segunda lengua. Algunos autores recomiendan la preparación minuciosa, paciente de tarjetas de uso rápido (FLASHCARDS) en la tarea de autoayuda para aprender y retener unas 1000 palabras nuevas. Se escribe en las tarjetas, la palabra inglesa y su representación fonética en un lado y el significado en el otro, luego en los momentos libres se sacan las tarjetas al azar y lo que el usuario debe hacer es decir de memoria el contenido del lado que no se ve. Los resultados según referencias varias y experiencia personal, son bastante satisfactorios.
Por todo lo anterior y minimizando obstáculos y limitaciones, se propone una versión computarizada del método FLASHCARDS que se cree debe ser de valiosa ayuda para todos los interesados en lograr dominio del inglés como segunda lengua en periodo relativamente corto, con la comodidad y rapidez de las computadoras actuales y, por sobre todo, con poco consumo de tiempo por sesión de práctica.

Los objetivos del presente trabajo son:

1. Aumentar el vocabulario de inglés intermedio de modo rápido.

2. Contribuir a la capacitación docente y estudiantil para su elegibilidad para becas en idioma inglés.

Es importante señalar que una de las motivaciones principales para la realización de este trabajo fue el llamado directo que hiciera el Dr. Chirinos, presidente de Concytec a aquellos docentes que habian tenido experiencia en paises de habla inglesa, para ayudar a otros colegas a superar la traba del idioma que era aún una causa de pérdida de las becas en oferta.

\section{EL PELADO QUIMICO DEL JUREL CONGELADO CON BAÑO EN SOLUCIÓN ALCALINA DE HIDRÓXIDO DE SODIO}

\author{
Luis Munante Angulo, Walter Ibárcena \\ Fernandez. \\ Facultad de Ing. Pesquera
}

El presente trabajo de investigación trata de determinar los parámetros óptimos en el despellejado químico del pescado, utilizando una solución de Hidróxido de Sodio.

La especie utilizada es el Jurel, Trachurus murphyi, al estado congelado. En primer lugar se realizó el análisis físico organoléptico para evaluår su calidad, a continuación fue sometido a baño en una solución de Hidróxido de Sodio, temperatura de solución y periodos de tiempo de inmersión del pescado, que tienen efectos sobre el despellejado y la calidad del pescado. El producto pelado quimicamente es sometido a una calificación para evaluar el grado de despellejamiento y la condición de la textura del tejido muscular, a través de una tabla de calificación adecuada y adoptada para el presente trabajo.

El flujo óptimo del despellejamiento quimico del Jurel es el siguiente: Materia prima congelada (Entera) -Lavado superficial- descabezado, 
eviscerado, sin alea caudal -lavado con agua potableevaluación sensorial. El producto final presentó la siguiente composición química proximal: humedad $75,69 \%$; proteinas $19,96 \%$; lipidos $2,01 \%$; cenizas $1,40 \%$ y carbohidratos $0,40 \%$. Los constituyentes de pescado pelado quimicamente en comparación con la materia prima no sufrieron variaciones significativas.

Se prepararon dos muestras: una muestra obtenida del producto pelado químicamente y otra muestra del pelado manual, en las mismas condiciones (Cocción a $100^{\circ} \mathrm{C}$ por 30 minutos) y se ha presentado a 10 panelistas quienes evaluaron sensorialmente el olor, sabor y apariencia, utilizando una escala de puntos. Luego se procedió a un análisis estadístico (ANVA), detectándose que no existia diferencias significativas entre ambas muestras.

El producto final obtenido, desde el punto de vista sensorial, es de buena calidad y apto para el consumo humano.

\section{EVALUACIÓN DE LOS PRINCIPALES RECURSOS HIDROBIOLOGICOS MARINOS DEL SUR DEL PERÚ}

Nelver Coronel Flores.

Facultad de Ing. Pesquera

En el presente trabajo, concluido en la primera parte, se realiza un análisis de la información estadistica de la comercialización o abastecimiento, al estado fresco y destinado al consumo humano directo, de los productos hidrobiológicos en la ciudad de Tacna, en base a registros de los últimos 10 años (1984-1993). Se determina que dentro de estos recursos, el pescado es el que ocupa el primer lugar, con aproximadamente el $80 \%$ del volumen total: la diferencia es cubierta por los denominados "mariscos". Dentro del recurso pescado, son la "cojinova" Seriorella vielacea, "bonito" Sarda chilensis, "Iorna" Sciaena deliciosa, "jurel" Trachurus symmetricas murphyi, "caballa" Scomber japonicus peruanis, "lisa" Mgil sp, "sargo" Anisotremus scapularis y "tollo" Mustelus dorsalis, en ese orden, los que ostentan los mayores volúmenes, pues en conjunto representan aproximadamente el $85 \%$ del total comercializado. Dentro de los mariscos, es la "macha" Mesodesma donacium la que ocupa el primer lugar, seguida del "choro" Aulacomya ater y "almeja" Protothaca thaca, pues en conjunto representan aproximadamente el $75 \%$ del volumen total.

\section{PROCESAMIENTO METALÚRGICO DE MINERALES SEMI-OXIDADOS DE COBRE-MINA IVON-ITE}

\author{
Nataniel Linares Gutiérrez, Rubèn C'anaviri LL. \\ Facultad de Ing. Metaliurgica
}

El mineral de cobre oxidado con una ley de $1,400 \%$ Cu y $8,0 \mathrm{~g} \mathrm{Au} / \mathrm{t}$ se ha sometido al proceso de flotación por espumas mediante el método de sulfidización, que consiste en sulfidizar la pulpa que tiene una granulometria del $62 \%$-m200 con una cantidad de $800 \mathrm{~g} / \mathrm{t}$ de sulfuro de sodio y a un $\mathrm{pH}$ de 8 , cuyas condiciones permiten obtener un concentrado conteniendo $18,85 \% \mathrm{Cu}$ y $200,2 \mathrm{~g} \mathrm{Au} / \mathrm{t}$ y recuperaciones que van del $51 \%$ para el cobre y del $80 \%$ para el oro. Con estos resultados se puede considerar viable el proceso propuesto para el procesamiento de este mineral.

Cuando se tiene un mineral cuyo elemento valioso está como óxido (incluyendo carbonatos, silicatos, etc), el procedimiento de extracción más adecuado es la lixiviación ácida o en algunos casos alcalina; sin embargo, cuando contiene además un metal precioso como el oro, el procesamiento hidrometalúrgico se complejiza, haciendo la extracción de los dos metales más complicado. Una alternativa de solución a este problema es el de concentrar ambos metales valiosos en un concentrado por el método de flotación por espumas de no-sulfuros, para lo cual la concentración puede lograrse por:

- Flotación de óxidos con ácidos grasos.

- Flotación por sulfidización.

Siendo este último el seleccionado para nuestro estudio.

\section{CONSTRUCCIÓN DE MEZCLADORES- SEDIMENTADORES PARA SISTEMAS DE EXTRACCIÓN POR SOLVENTES A NIVEL DE LABORATORIO}

\section{Nataniel Linares Gutiérrez. \\ Facultad de Ing. Metalúrgica}

Se ha desarrollado un programa de laboratorio para el estudio de la extracción del cobre por solventes orgánicos. Las pruebas realizadas de acuerdo a este procedimiento, que consiste en el paso de un soluto o especie metálica, disuelto en una fase acuosa a otro liquido -con el objeto de separarla de otras especies de la solución (EXTRACCIÓN) y la recuperación de la especie extraida al efectuar el 
proceso inverso (REEXTRACCION)-, han demostrado que es factible lograr la recuperación de cobre operando celdas experimentales de evaluación y la confirmación de las condiciones de operación predeterminadas a partir del estudio de las isotermas de equilibrio.

El presente documento resume las consideraciones iniciales de la quimica del sistema planteado, los criterios básicos de diseño y construcción empleados para la instalación de una celda experimental y un estudio de las caracteristicas de extracción y reextracción del reactivo ACORGAPT5050 a nivel de laboratorio. Considerando que nuestro objetivo último fue el de diseñar y construir un circuito piloto de extracción por solventes, se hace especial mención sobre la manera como se ha conceptuado la construcción de los diagramas de McCabe Thiele. El resultado es el diseño de un circuito constituido de una etapa de extracción y una etapa de reextracción.

En enero de 1993 se inició en los laboratorios de la Facultad de Ingenieria Metalúrgica de la UNJBG de Tacna, un programa para el estudio del proceso de EXTRACCION POR SOLVENTES. Dentro de IOS objetivos de este estudio se ha considerado la construcción de unidades mezcladoras sedimentadoras y la operatividad de los mismos en un sistema continuo, en el desarrollo de un proyecto de investigación integral, con miras a definir las alternativas de tratamiento de los minerales de la SubRegión de Tacna. Entonces se delineó un programa de estudio por el cual, primeramente, debiamos recopilar toda la información técnica disponible para mejorar y estandarizar los métodos analíticos y reducir, por tanto, el número de pruebas metalúrgicas y los costos de la investigación. En segundo lugar, los ensayos preliminares a nivel de vasos agitados debian confirmar las especificaciones técnicas encontradas para el reactivo extractante y hacer factible la determinación de las isotermas de equilibrio que permitan diseñar el sistema continuo propuesto. Por último, la construcción de un número de celdas experimentales sería necesario para el desarrollo de prucbas de optimización del proceso y la ejecución de pruebas continuas de evaluación de las condiciones de operación pre-determinadas.

Los ensayos preliminares de extracción fueron ejecutados sobre soluciones sintéticas y los de optimización del proceso sobre soluciones acuosas de lixiviación diluidas, de acuerdo a los diseños experimentales, factorial y hexagonal, hasta encontrar los valores óptimos de las variables operacionales seleccionadas.

\section{RENDIMIENTO DE CRISTALIZACIÓN DEL SO4Cu- 5H20 A TRAVÉS DE CÁTODOS DE PLOMO}

\author{
Luis Caso Palpa, José Luis Tenorio. \\ Facultad de Ing. Metalurgica
}

El presente trabajo, "Rendimiento de cristalización del So4Cu. $5 \mathrm{H} 2 \mathrm{O}$ a través de cátodos de plomo", se hizo a partir de una solución producida por lixiviación con ácido sulfúrico diluido de una columna de óxidos de cobre, y a partir de soluciones sintéticas similares a las condiciones en que se trabaja en la planta del sulfato de la Cía. Pativilca -esto como una alternativa de dar mayor valor agregado a los minerales oxidados de cobre, existentes en la zona andina de Tacna-

El estudio está orientado a realizar pruebas de rendimiento de cristalización a partir de un diseño experimental donde el rendimiento o producción de cristales en menor tiempo es evaluado desde 2 aspectos: velocidad de formación de núcleos y velocidad de crecimiento de núcleos, con láminas de plomo-antimonio como agente nucleante.

Se realizaron las diferentes pruebas experimentales utilizando el diagrama de solubilidad (Isoterma de solubilidad) del sulfato de cobre en solución de ácido sulfúrico y considerando un rango de temperaturas para la zona estudiada entre $10^{\circ} \mathrm{C}$ a $18^{\circ} \mathrm{C}$, donde se evaluaron los efectos de la calidad de la solución madre, nivel de sobresaturación de cobre, concentración de ácido sulfúrico, tipo de enfriamiento, tipo de acidificación, agitación y agentes nucleantes. Los resultados se evaluaron midiendo la recuperación o rendimiento de cristalización.

\section{DIAGNÓSTICO DE ALGUNAS CARACTERISTICAS PSICO-SOCIALES EN UN GRUPO DE INDIVIDUOS QUE HAN MANIFESTADO CONDUCTAS DELICTIVAS}

Carlos Pauca Lazo.
Facultad de Ciencias Administrativas

La investigación descrita se ha orientado a obtener mayor conocimiento acerca de la conducta delictiva, considerando a ésta como una de las tantas variantes por las cuales se expresa el psiquismo humano ante causas y motivos existentes en la realidad objetiva que rodea al hombre.

La personalidad y la conducta constituyen la esencia y fenómeno del comportamiento humano; es 
por ello que a través de este trabajo de investigación nos hemos propuesto como principal objetivo hacer una descripción de algunas caracteristicas psicosociales de la personalidad de un grupo de individuos que han delinquido y que se encuentran internados en el penal de Pocollay - Tacna, a fin de proponer acciones de prevención, concretamente sobre el delito.

"La orientación profiláctica de la labor de los organismos que luchan contra la delincuencia, obliga no sólo a prevenir la delincuencia en conjunto, sino también a impedir los delitos; es decir, a actuar antes de que un hombre aprenda la via criminal. Esto es precisamente lo que hace necesario preveer la conducta de algunos individuos, los que han sido sometidos anteriormente a responsabilidad criminal, asi como de las personas sin antecedentes penales. La previsión de la posible conducta del individuo, sobre todo de la conducta anti-social, es una tarea dificil. Sin embargo las dificultades pueden ser superadas mediante un estudio de la personalidad, del mecanismo de conducta del hombre, mediante una investigación minuciosa de los factores exteriores $\mathrm{e}$ interiores que actuan sobre el individuo y su conducta. Se evalúa precisamente al individuo, su conducta en el futuro"

El delito es un fenómeno causal, de naturaleza psicológica que obedece a la ley de la posibilidad y la realidad cuyas causas y motivos se encuentran donde interactúa el hombre. Esta tesis descarta en forma definitiva algunas afirmaciones en el sentido de que el delito es determinado por el medio social, por el contenido instintivo o por algunos condicionamientos biológicos del individuo.

El delito significa una serie de actos que causan perjuicio material y espiritual a la sociedad. La comprensión del daño que ocasiona este fenómeno nos conduce a valorar la importancia que tiene su tratamiento, especialmente para su prevención.

\section{DIAGNOSTICO DE LA PEQUEÑA Y MICRO EMPRESA EN TACNA. ASPECTOS ADMINISTRATIVOS Y DE GESTIÓN}

Alejandro Mego Cubax.

fincultud de Ciencias telministronivas

El presente trabajo tiene por finalidad conocer la situación actual de los problemas que los pequeños y micro empresarios tienen en la gestión empresarial; y de esta manera tener criterios que permitan elaborar planes de asistencia técnica, brindar capacitación y asesoramiento que reviertan la situación de crisis por la que atraviesan en la actualidad.

El trabajo comprende cuatro capitulos, de los cuales el primero trata de definir qué es una pequeña y micro empresa, señalando sus caracteristicas, particulares en lo referente a su conducción, financiamiento, base legal y tributario

En el segundo formulamos el diagnóstico administrativo y de gestión en base al análisis de las encuestas realizadas, lo que nos permite conocer aspectos generales de las empresas y negocios; asi como, la forma legal de constitución. inversión promedio y el número de empresas que operan en el sector comercio e industria.

Por otra parte se contempla el tipo de gestión que realizan en las áreas funcionales de ventas, abastecimiento, producción, finanzas y vinculación al sistema. Además se estudia todo lo concerniente al nivel de empleo, asesoramiento y capacitación que requieren los pequeños y micro empresarios de Tacna.

En los capitulos tres y cuatro se presentan las conclusiones más importantes; se explica la metodologia que se ha utilizado en la elaboración del presente trabajo de investigación.

Consideramos, finalmente, que el estudio realizado debe servir para formar criterios adecuados en la universidad y en especial en la Facultad de Ciencias Administrativas, como base para la implementación de asistencia técnica, capacitación y asesoramiento a los pequeños y micro empresarios de Tacna, como parte de la labor de proyección social a la comunidad.

MAGNITUD DE LA HIPERTENSIÓN ARTERIAL Y ALGUNOS FACTORES QUE LA CONDICIONAN EN LA POBLACIÓN DE 15 A 64 AÑOS DE EDAD EN EL DISTRITO DE TACNA

\author{
Maria Vargas Salecdo, Elide Tipacti \\ Sotomayor: \\ Fincultad de Einfermeria
}

Es cada vez más frecuente la preocupación que manifiesta la población por el incremento de los casos de hipertensión arterial y otras enfermedades cardiovasculares, sus causas y consecuencias; pero la información actualmente disponible sobre el tema es limitada y por lo tanto, no se puede contar con indicadores sobre la naturaleza y proyección del problema. 
Es cierto que la preocupación por contar con bases sólidas para conocer y combatir el problema, nació hace unos años atrás cuando esfuerzos aislados procuraron responder algunas interrogantes sobre la situación y/o factores que intervienen en su presentación.

A pesar de que, desde la década 50 , se sabía que la hipertensión arterial intervenia en el momento de la morbilidad cardiovascular en los paises desarrollados, fueron los estudios efectuados en las décadas 60 y 70 los que claramente mostraron la relación entre hipertensión y las muertes por complicaciones vasculares en los órganos blanco: corazón, cerebro, riñón y vasos sanguíneos.

En la última década se produjo un gran estímulo a la investigación en aspectos epidemiológicos y básicos, tales como mecanismos fisiopatológicos. Se avanzó en la búsqueda de mejores fármacos antihipertensivos, diseño de tratamientos y seguimiento de pacientes.

Numerosas encuestas de prevalencia, incluyendo algunas en América Latina, mostraron la magnitud del problema y las dificultades en la detección precoz y tratamiento adecuado.

Estos fenómenos están relacionados causalmente con el aumento de la frecuencia de enfermedades crónicas y entre éstas las cardiovasculares ocupan un lugar prioritario.

En el Perú, la situación de la hipertensión arterial medida en términos de magnitud y factores condicionantes es limitada y se estima que existe entre
5 a $11 \%$ de la población que presenta hipertensión del tipo diastólica en zonas a nivel del mar.

Específicamente en Tacna, se desconoce la magnitud de ésta, donde la incidencia de enfermedades y/o complicaciones cardiovasculares se va incrementando dia a dia, constituyendo la causa de mortalidad general en la población adulta.

Después de analizar e interpretar los datos arribamos a las siguientes conclusiones:

1. Que las cifras obtenidas se refieren a hipertensión arterial causal registrada en un momento cualquiera, y no las que pudieran obtenerse en condiciones adecuadas de reposo, ayuno, temperatura ambiente y ausencia de estimulos emocionales.

2. Que la H.T.A. se presenta con mayor frecuencia en adultos cuyas edades fluctúan entre 45 a 64 (47\%) años de edad, que en su mayoría son de la zona urbana y periurbana.

3. La AD se presenta con mayor incidencia en adultos de ambos sexos de 55 a 64 años de edad, teniendo el mayor porcentaje el sexo femenino.

4. De acuerdo a los datos obtenidos el mayor porcentaje de hipertensos que tienden a subir de peso se encuentran en el sexo femenino con el $31 \%$.

5. El $27 \%$ de los hipertensos sufren alteración o complicación funcional cardiovascular; mientras que sólo un $16 \%$ sufre complicación renal. 


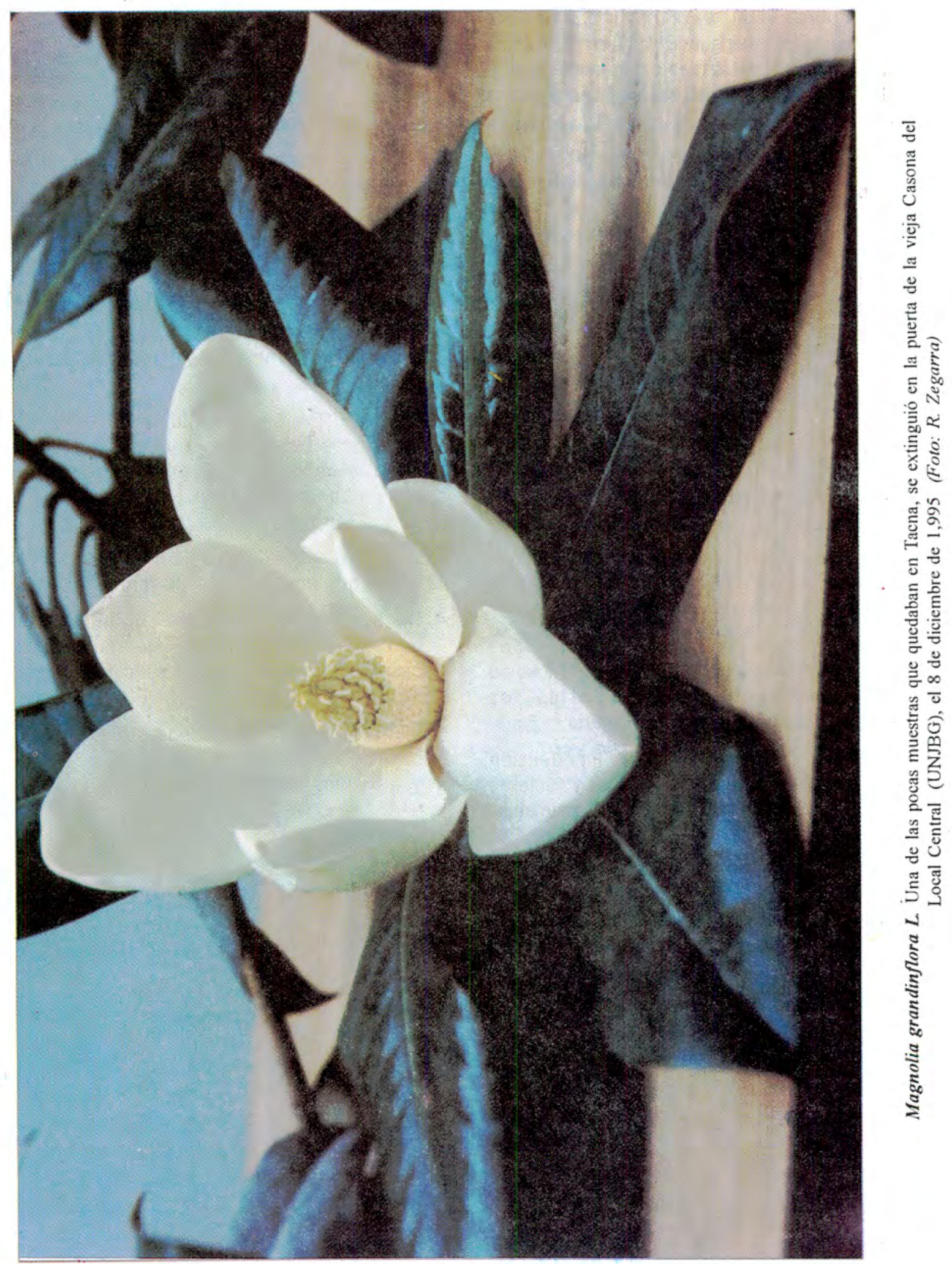

